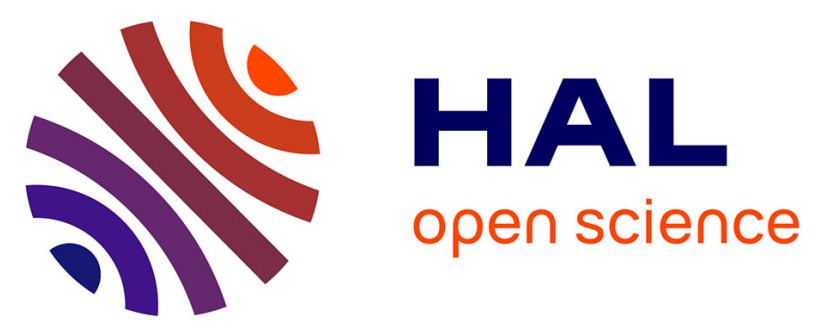

\title{
Compact optoelectronic oscillator using whispering gallery mode resonators for radio-frequency and millimeter wave generation
}

K. Volyanskiy, P. Salzenstein, H. Tavernier, M. Pogurmirskiy, Y.K. Chembo, L. Larger

\section{To cite this version:}

K. Volyanskiy, P. Salzenstein, H. Tavernier, M. Pogurmirskiy, Y.K. Chembo, et al.. Compact optoelectronic oscillator using whispering gallery mode resonators for radio-frequency and millimeter wave generation. SPIE OPTO, Jan 2011, San Francisco, United States. pp.79360B, 10.1117/12.876985 . hal-00655135

\section{HAL Id: hal-00655135 \\ https://hal.science/hal-00655135}

Submitted on 4 May 2021

HAL is a multi-disciplinary open access archive for the deposit and dissemination of scientific research documents, whether they are published or not. The documents may come from teaching and research institutions in France or abroad, or from public or private research centers.
L'archive ouverte pluridisciplinaire HAL, est destinée au dépôt et à la diffusion de documents scientifiques de niveau recherche, publiés ou non, émanant des établissements d'enseignement et de recherche français ou étrangers, des laboratoires publics ou privés. 


\title{
Compact optoelectronic oscillator using whispering gallery mode resonators for radio-frequency and millimeter wave generation
}

\author{
Kirill Volyanskiy ${ }^{1}$, Patrice Salzenstein ${ }^{2}$, Hervé Tavernier $^{1}$, Maxim Pogurmirskiy ${ }^{3}$, \\ Yanne K. Chembo ${ }^{1}$ and Laurent Larger ${ }^{1}$ \\ 1 FEMTO-ST Institute (CNRS UMR6174), Optics Department, \\ 16 Route de Gray, 25030 Besançon cedex, France \\ ${ }^{2}$ FEMTO-ST Institute (CNRS UMR6174), Time-Frequency Department, \\ 26 Chemin de l'Epitaphe, 25030 Besançon cedex, France \\ 3 National Research University of Information Technologies, Mechanics and Optics (ITMO), \\ Saint-Petersburg, Russia.
}

\begin{abstract}
Optoelectronic oscillators are ultra-pure microwave generators based on optical energy storage instead of high finesse radio-frequency resonators. We present in this communication a new and compact architecture where the optical energy storage is performed by trapping laser light into the ultra-high $Q$ whispering gallery modes of a millimeter-size disk resonator. As a proof of concept, we demonstrate the generation of a $10.7 \mathrm{GHz}$ microwave with a phase noise of $-110 \mathrm{dBrad}^{2} / \mathrm{Hz}$ at $100 \mathrm{kHz}$. We also discuss in detail the potential of this architecture for the generation of microwaves with a frequency ranging from 50 to $200 \mathrm{GHz}$.
\end{abstract}

Keywords: Optoelectronic oscillators, microwave generation, terahertz generation, whispering gallery modes.

\section{INTRODUCTION}

Optoelectronic oscillators (OEOs) are ultra-pure microwave generators based on optical energy storage instead of high finesse radio-frequency $(\mathrm{RF})$ resonators. ${ }^{1}$ These oscillators have many specific advantages, such has exceptionally low phase noise, and versatility of the output frequency which is determined by the RF bandwidth of the optoelectronic components. Such ultra-pure microwaves are indeed needed in a wide range of applications, including time-frequency metrology, frequency synthesis, and aerospace engineering.

Generally, the optical storage element in OEOs is an optical fiber delay line, and the output microwave frequency of the system is defined by a narrow RF band-pass filter in the electronic segment of the feedback loop. This original configuration yields excellent phase noise performance, but it also has several drawbacks. The first one is that the optical delay line is bulky, so that the oscillators can not be considered as an optimal transportable microwave source. Along the same line, this bulky delay line element has to be temperaturestabilized, a feedback control process which is energy greedy. Finally, the fiber delay line generates spurious peaks very close to the carrier (few tens of $\mathrm{kHz}$ ), which are highly detrimental in several applications.

An alternative to circumvent all these drawbacks is to replace the optical fiber delay line by an ultra-high $Q$ whispering gallery-mode (WGM) optical resonator. ${ }^{2}$ In this case, the microwave oscillation frequency is defined by the free-spectral range (FSR) of the resonator, while energy storage is performed by trapping laser light into the ultra-low loss WGMs. This configuration provides an interesting solution to the problems raised above, as the same element (WGM resonator) at the same times defines the oscillating frequency and ensures the energy storage. In particular, these WGM optoelectronic oscillators are compact, they do not generate delay-induced spurious peaks in the RF spectrum, and they are compatible with compact temperature control system, since

Further author information: (Send correspondence to Y. K. C.)

E-mail: yanne.chembo@femto-st.fr ; Telephone: +33 381666401. 


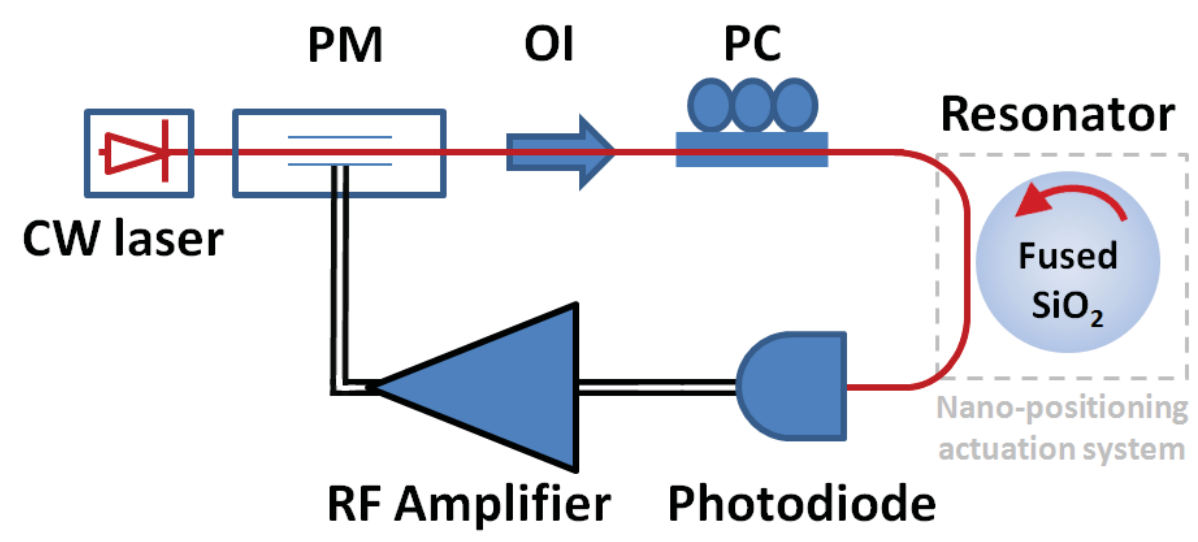

Figure 1. Experimental setup of the compact OEO. CW: continuous-wave; OI: optical isolator; PC: polarization controller; $\mathrm{PM}$ : phase modulator; $\mathrm{RF}$ : radio-frequency; $\mathrm{SiO}_{2}$ : silica.

it is limited to a much smaller volume. This ultra-pure microwave source thereby becomes easily transportable. This is a highly desirable feature in many applications, such as in aerospace engineering for example.

The other advantage of this new configuration is the use of phase modulation instead of intensity modulation. Effectively, intensity modulators are environment sensitive devices, which is not desired when high device stability is required for pure tone generation. On the contrary, phase modulation involves a differential phase-to-intensity conversion, which is not sensitive to electro-optic drifts. The fact that a resonator is an imbalanced interferometer is precisely the situation required for the differential phase-to-intensity conversion. This originates from standard differential optical phase modulation techniques, which are known in optical communications to offer superior performances as soon as the modulation speed is very high.

We present in this communication a new configuration where a microwave is generated using a single-loop OEO with ultra-high Q WGM disk resonator. In the next sections, we present in detail the experimental setup of this OEO, present the experimental results on phase noise measurement, and discuss its potential for mm-wave systems.

\section{THE EXPERIMENTAL SYSTEM}

The OEO under study is presented in Fig. 1. This architecture is reminiscent of the original configurations of single-loop OEOs. Here, the main differences are that the fiber delay line has been replaced by a WGM resonator, while the Mach-Zehnder modulator has been replaced by a phase modulator. The fused silica optical resonator has a $Q$ factor of $10^{8}$, and diameter $\sim 5 \mathrm{~mm}$, yielding a FSR of $10.7 \mathrm{GHz}$. The phase modulator has a half-wave voltage $V_{\pi}=2.8 \mathrm{~V}$, and it is driven by a $1550 \mathrm{~nm}$ semiconductor laser (RIO Orion laser module), internally stabilized with a precision of $2 \mathrm{pm}$. The phase-modulated laser beam is first polarization-controlled in order to select TE or TM modes, and then critically coupled into the resonator through a tapered fiber. In other words, the intrinsinc $\left(Q_{\text {int }}\right)$ and extrinsic $\left(Q_{\text {ext }}\right)$ quality factors are set to be equal. The difference of optical index between fused silica $(=1.44)$ and air $(\simeq 1)$ enables the internal reflection inside the resonator. Finally, the optical output signal of the tapered fiber is detected with a photodiode (DSC30S). A transduced microwave is obtained, and is used to close the feedback loop in the RF input of the phase modulator after amplification (AML 218L4401 driver with a $42 \mathrm{~dB}$ gain).

A major source of losses in the optical cavity is surface roughness and degradation through water vapor pollution. We have also significantly improved the coupling efficiency with the use of a $1 \mathrm{~nm}$ resolution nanopositioning system. Figure 2 represents this positioning system with tapered fiber and our disk-resonator. The tapered fiber is coupled to the fused silica disk-resonator. White light illumination is only for monitoring of the coupling zone via a video camera, which is helpful for the preliminary rough positioning of the taper close to the disk resonator. We can thereby monitor how close are the fiber and the resonator. The nano-positioning system provides enough space for movement in a $12 \times 12 \mathrm{~mm}^{2}$ surface. 


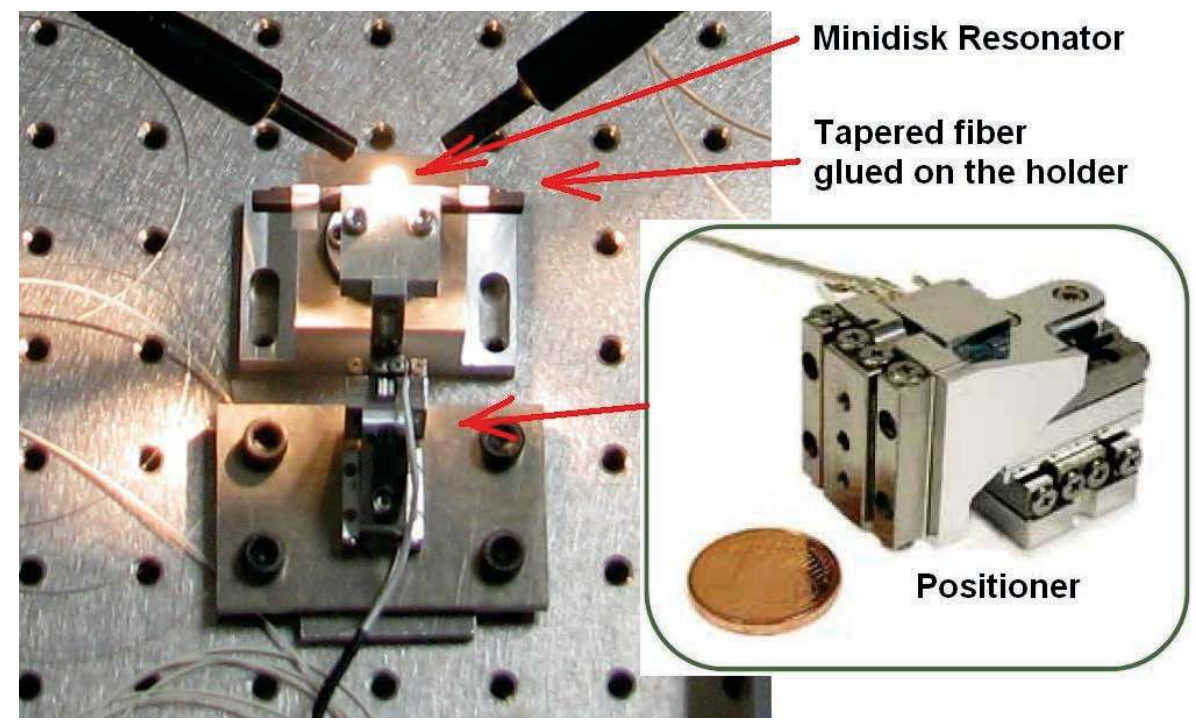

Figure 2. Picture of the coupled resonator, using a nano-positioning system.

A picture of the whole oscillator is represented in Fig. 3. In our laboratory experimental setup, the WGM OEO is quite compact and easily fits into the $\mathrm{A}_{3}$ format $\left(297 \times 420 \mathrm{~mm}^{2}\right)$. Optimized packaging could certainly reduce this size by a factor of ten, and even more if integrated photonics solutions are considered.

\section{EXPERIMENTAL RESULTS}

Figure 4 presents a typical phase noise spectrum measurement from our OEO. The phase noise was measured for a frequency offset ranging from $10 \mathrm{~Hz}$ to $100 \mathrm{kHz}$ using a dedicated optoelectronic phase noise measurement bench, developed in our laboratory. ${ }^{3}$ As theoretically predicted, the microwave has a frequency equal to the disk-resonator FSR $(10.7 \mathrm{GHz})$, and an output power of $1.6 \mathrm{dBm}$. The phase noise performance is evaluated to $-90 \mathrm{dBrad}^{2} / \mathrm{Hz}$ at $10 \mathrm{kHz}$ from the carrier, and $-110 \mathrm{dBrad}^{2} / \mathrm{Hz}$ at $100 \mathrm{kHz}$. This level of the phase noise can be significantly improved, for example through temperature stabilization, laser frequency-locking with respect to the absolute resonance frequency of the disk, or enhanced isolation from environmental vibrations. In fact, our OEO was built as a proof-of-concept oscillator and the topology has not been optimized. We expect that careful packaging and isolation from environmental vibrations would significantly increase the phase noise performance of this oscillator.

\section{POTENTIAL FOR MM-WAVE GENERATION}

An open point is the suitability of this system for ultra-low phase noise mm-wave generation. Nothing theoretically prevents the systems to oscillate at such frequencies.

Experimentally, the main problem arises from the bandwidth of the optoelectronic components within the loop. Photodiodes with a bandwidth around $100 \mathrm{GHz}$ are already commercially available. Moreover, The technology for photodiodes with bandwidths at the edge of mm-waves $(\sim 300 \mathrm{GHz})$ is nowadays available at the stage of prototypes. On the other hand, early prototypes of RF amplifiers with a $200 \mathrm{GHz}$ bandwidth are also available. As far as integrated modulators are concerned, ultra-high density optical communication systems are fueling the demand for devices able to handle bit rates of $160 \mathrm{~Gb} / \mathrm{s}$, and in the next decade, the needs will probably be set to $320 \mathrm{~Gb} / \mathrm{s}$. In consequence, we can realistically expect that in a near future, the elements needed for ultra-low phase noise mm-wave generation will be standard components and commercially available. It is also noteworthy that generating frequencies in the THz range require even smaller WGM resonators (micrometer size), and that they require other technologies for both fabrication and monitoring. 


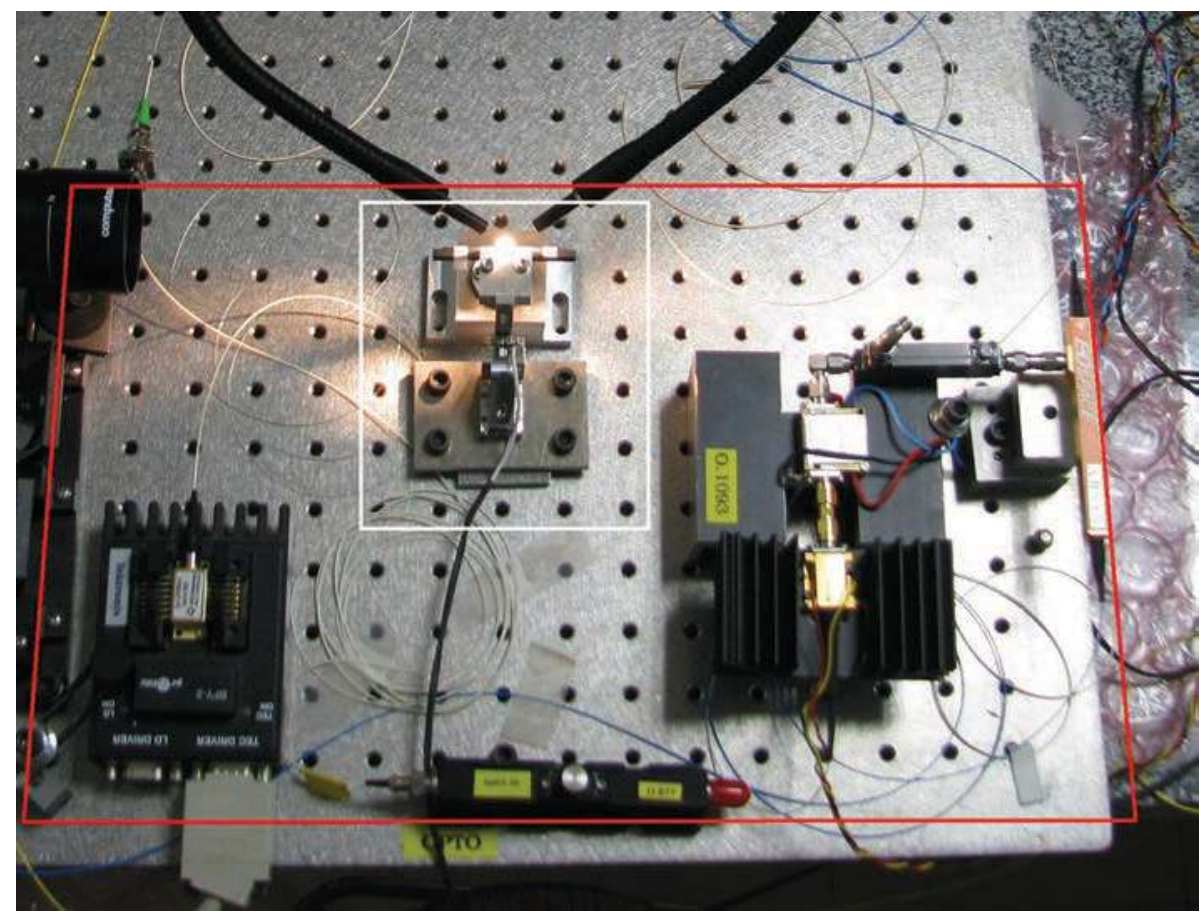

Figure 3. Picture of the whole oscillator. The large (red) rectangle represents the $\mathrm{A}_{3}$ format $\left(297 \times 420 \mathrm{~mm}^{2}\right)$, while the small (white) rectangle represents the coupled resonator as displayed in Fig. 2.

\section{CONCLUSION}

We have reported a new OEO architecture based on WGM resonators and phase modulation. This work contributes to show the high potentiality of optical millimeter-size resonator for high performance, compact and low consumption microwave generators. We have been working in the X-band for our demonstration but it could be interesting to synthesize significantly higher frequencies, at the edge of the mm-band. A particularly interesting feature is that this configuration is compatible with chip integration, and could therefore be a transportable source for ultra-pure microwave generation. From a purely scientific point of view, there are also several open issues will have to be investigated in detail, like for example the effect intrinsic nonlinear phenomena (Kerr, Raman, Brillouin, etc.). There is also a need for a deterministic model, later completed with a stochastic counterpart, in order to investigate the dynamical and phase noise properties of the system ${ }^{4,5}$ These investigations will be the focus of future work.

\section{ACKNOWLEDGMENTS}

The authors acknowledge financial support from the Centre National d'Etudes Spatiales (CNES).

\section{REFERENCES}

[1] X. S. Yao and L. Maleki, "Optoelectronic microwave oscillator," J. Opt. Soc. Am. B 13, 1725-1735 (1996).

[2] A. B. Matsko, L. Maleki, A. A. Savchenkov, and V. S. Illchenko, "Whispering gallery mode based optoelectronic microwave oscillator," J. Mod. Opt. 50, 2523-2542 (2003).

[3] K. Volyanskiy, J. Cussey, H. Tavernier, P. Salzenstein, G. Sauvage, L. Larger, and E. Rubiola, "Applications of the optical fiber to the generation and measurement of low-phase-noise microwave signals," J. Opt. Soc. Am. B 25, 2140-2150 (2008).

[4] Y. K. Chembo, L. Larger, H. Tavernier, R. Bendoula, E. Rubiola and P. Colet, "Dynamic instabilities of microwaves generated with optoelectronic oscillators," Opt. Lett. 32, 2571-2573 (2007). 


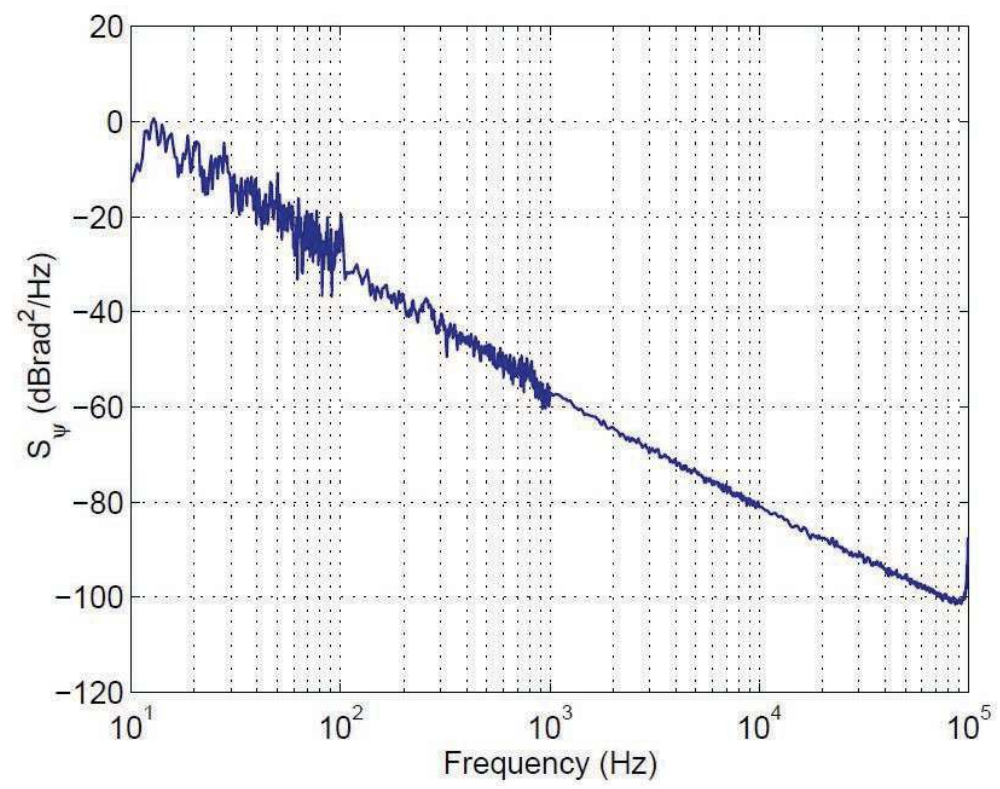

Figure 4. A typical phase noise spectrum of the WGM OEO with phase modulation. The microwave has a frequency of 10.7 $\mathrm{GHz}$ and an output power of $1.6 \mathrm{dBm}$. The phase noise performance is $-90 \mathrm{dBrad}^{2} / \mathrm{Hz}$ at $10 \mathrm{kHz}$ from the carrier, and $-110 \mathrm{dBrad}^{2} / \mathrm{Hz}$ at $100 \mathrm{kHz}$.

[5] Y. K. Chembo, K. Volyanskiy, L. Larger, E. Rubiola and P. Colet, "Determination of phase noise spectra in optoelectronic microwave oscillators: a Langevin approach," IEEE J. of Quantum Electron. 45, 178-186 (2009). 